\title{
Water-Insoluble Particles in Spring Snow at Mt. Tateyama, Japan: Characteristics of the Shape Factors and Size Distribution in Relation with Their Origin and Transportation
}

\author{
Jingmin LI and Kazuo OSADA \\ Graduate School of Environmental Studies, Nagoya University, Nagoya, Japan \\ (Manuscript received 6 September 2006, in final form 11 December 2006)
}

\begin{abstract}
The number-size distribution and shape factors of water-insoluble particles (WIP) in four dirty snow layers in the spring of 2001 at Mt. Tateyama, Japan, were analyzed using scanning electron microscopy, and using optical microscopy with a digital camera. Results show that the median aspect ratio (ratio of the longest dimension $b$ to the orthogonal width $a ; b / a)$ of the WIP varied within 1.22-1.31. Only a few particles with an aspect ratio of more than 2.5 were observed. The median circularity factor $\left(4 \pi S / l^{2} ; S\right.$ is projection area, and $l$ is periphery length) varied from $0.83-0.97$.

Combined with backward air trajectories, visibility reducing surface weather reports, additional results of rain containing Saharan dust and dry deposition samples, the number and shape factor distributions versus particle size were characterized as (1) narrower distributions of number-size and shape factors for the Saharan dust, and (2) bi-modal number-size distribution for dry deposited dust. Results suggest that the proportions of nearly spherical (higher circularity nearly 1) and less elongated (lower aspect ratio close to 1) particles were higher in the sample that had been transported a longer distance from the dust source areas.
\end{abstract}

\section{Introduction}

Atmospheric mineral dust particles play an important role in atmospheric radiation transfer (Sokolik and Toon 1996; Sakai et al. 2000, Intergovernmental Panel on Climate Change (IPCC) 2001), and the geochemical cycle of atmospheric constituents (Duce et al. 1980; Uematsu et al. 1983; Arimoto et al. 1985). The shape and size distributions of mineral dust aerosols are important parameters to deduce their optical and aerodynamic properties. Assuming that spherical particles represent mineral dust aerosols might introduce errors in ra-

Corresponding author: Jingmin Li, Graduate School of Environmental Studies, Nagoya University, Furo-cho, Chikusa-ku, Nagoya 464-8601, Japan.

E-mail: lijm@stelab.nagoya-u.ac.jp

(C) 2007, Meteorological Society of Japan diative transfer calculation (Sokolik et al. 2001; Kahnert and Kylling 2004; Kahnert et al. 2005; Kahnert and Nousiainen 2006), might cause an underestimation of collection of particles for wet deposition (Volken and Schumann 1993), and might significantly affect the results of satellite and remote sensing retrieval (Masuda et al. 2002; Kalashnikova and Sokolik 2002; Astafyeva and Babenko 2004).

Mineral dust particles are known to have irregular shapes. For example, Okada et al. (1987) reported the median aspect ratio (ratio of the longest dimension $b$ to the orthogonal width $a ; b / a)$ of 1.4 for aerosol samples collected at Nagoya in Japan during Asian dust events, based on electron microscopic analyses of individual mineral dust particles. Later, Okada et al. (2001) studied the shape of mineral dust particles collected from three arid regions in China, and found a similar median aspect ratio 
of 1.4 for the samples. They reported a tendency for decreasing circularity factor $\left(4 \pi S / l^{2}\right.$; $S$ is the projection area and $l$ is the periphery length) with increasing particle diameter. Gao and Anderson (2001) also reported the circularity factor of Chinese aerosols collected in spring. Reid et al. (2003) suggested a median average aspect ratio of 1.9 for airborne particles from Africa collected at the Puerto Rico Dust Experiment (PRIDE) field site. The aspect ratio and circularity parameters, which describe the irregular shape of mineral dust particles, are eagerly anticipated to model radiative properties of the dust particles, but such data are currently very limited (Kalashnikova and Sokolik 2004).

Snow deposited in high mountains offers some advantages for the study of mineral dust particles in the atmosphere. Those advantages include: (1) snow as an archive of deposition during winter before snow melt; and (2) snow that is less contaminated by local dust and pollen than that from lowland areas. The air temperature at Murododaira (2450 m a.s.l.) of Mt. Tateyama (3015 $\mathrm{m}$ a.s.l.) rarely becomes higher than $0^{\circ} \mathrm{C}$ from late November through April. That sub-zero air temperature prevents snow melt. For that reason, deposited snow at Murododaira might provide a reliable snow archive. Reducing local dust contribution after snow deposition is also very important for analyzing long-range transport from distant source regions via the free troposphere.

The snow samples in this study were analyzed originally by Osada et al. (2004) for dust deposition processes and weight variation of water-insoluble particles (WIP). They preliminarily reported mono-modal and bi-modal volume-size distribution of water-insoluble particles, using a laser diffraction/scattering particle size distribution analyzer. However, the use of that method for sizing irregular and colored mineral dust particles might engender large uncertainty (Konert and Vandenberghe 1997; Naito et al. 1998). In this study, an image analysis using optical microscopy with a digital camera was applied, similar to that for the analysis by Yamamoto et al. (2004). Without relying on optical properties related to scattering and diffraction, image analysis under an optical microscope is comparable to that of a substance of known size. Image analyses also provides in- formation related to the shape factors of mineral dust particles.

This study is intended to provide data of aspect ratios and circularity factors of the dust particles in dirty snow samples obtained at Mt. Tateyama, and to elucidate the relationship of particle size with aspect ratios and circularity factors. We will also show the number-size distributions of super-micrometer dust particles in relation with deposition processes and source regions of mineral dust particles. Finally, the shift of shape factors and size parameters during long-range transport are discussed.

\section{Measurement}

\subsection{Sampling sites and snow sampling}

Mount Tateyama (3015 $\mathrm{m}$ a.s.1.), which has heavy snowfall during winter, is located in the northwest side of the Japanese Alps on Honshu Island (Fig. 1). Snow cover at Mt. Tateyama in spring is about $6-10 \mathrm{~m}$. Snow cover around the mountain area prevents wind-blown redistribution of local soil materials. As described in Osada et al. (2004), snow pits at three sites were dug for snow sampling: Midagahara

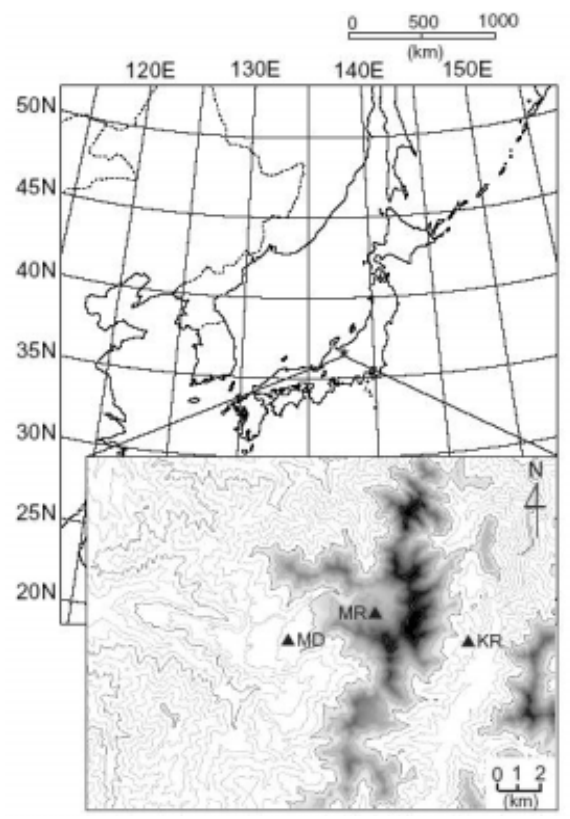

Fig. 1. Location of Mount Tateyama in Japan with three sampling sites: Midagahara (1830 $\mathrm{m}$, abbreviated as MD), Murododaira (2450 m, MR), and Kurobedaira (1780 m, KR). 
(1830 m, abbreviated as MD), Murododaira (2450 m, MR), and Kurobedaira (1780 m, KR). The sampling sites $\mathrm{MD}$ and $\mathrm{KR}$ are located $5 \mathrm{~km}$ distant from MR. These sites are suitable for continuous snow deposition because they are wide, flat areas that show similar temporal change of dust deposition during winter. For this study, remaining re-frozen samples that had been collected at Mt. Tateyama in spring 2001 were re-analyzed.

The vertical profiles of dust concentration in snow at MD, MR, and KR in 2001 are depicted in Fig. 2. Snow samples were taken from MR in March 2001, and KR and MD in April 2001. For higher dust concentration, we selected several snow layers marked as 1, 2, 3, and 4. For sample identification in this study, labels of MR-1, MR-2, MR-3, MR-4, MD-4, and KR-4 are used. According to the vertical profiles of the WIP at the three sites and local dirty snow observation near the mountain, the WIP in layer 4 (MR-4, MD-4, and KR-4) were considered to be deposited at the same event on 3 January 2001. These samples of layer 4 (MR- $4, \mathrm{MD}-4$, and $\mathrm{KR}-4$ ) were analyzed to confirm the horizontal variability of dust deposition, and veracity of this image analytical method.

The respective thicknesses of snow layers MR-1, MR-2, and MR-4 were greater than $10 \mathrm{~cm}$ in snow. As described in Osada et al.

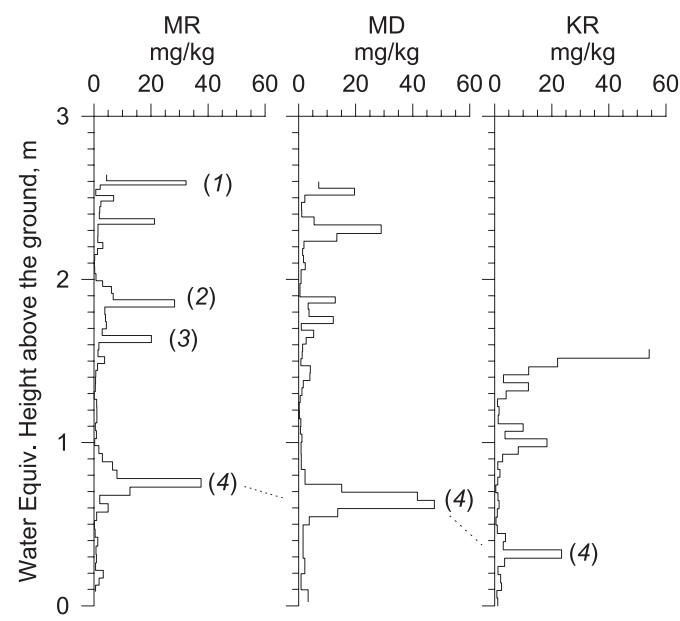

Fig. 2. Vertical profile of dust concentration in spring 2001 at MD, MR, and KR. Numbers with parentheses $(1,2,3,4)$ indicate dirty snow layers analyzed in this study.
(2004), these layers were considered to be formed by wet deposition. In contrast, the thickness of MR-3 was very thin (2 mm) with a sharp edge of the dirty layer, suggesting that the dust particles were deposited by a dry process on the snow surface, instead of wet deposition with snowfall. The dates of these dust events were inferred as 11 February for layer 2, 3 February for layer 3, and 3 January 2001 for layer 4 , based on local dirty snow observation near Mt. Tateyama, weather conditions, and the time variation of aerosol concentrations at Murododaira (Osada et al. 2004). The date of deposition for layer 1 was not specified using the matching method mentioned above.

\subsection{Reference samples}

In addition to deposited snow samples, a rain sample of 8 March 2005 at Toyama was also analyzed as a reference sample of the Saharan dust, as suggested by Park et al. (2005). Dirty rain samples (19 April 2006) and dry deposition samples (8 April 2006 and 24 April 2006) of Asian dust were collected using a polyvinyl chloride (PVC) bucket at the top of the building of the Graduate School of Environmental Studies, Nagoya University. The WIP of the dry deposition sample were collected after wetting using ultra-pure water.

According to visibility reducing surface weather reports (NRL/Monterey Aerosol Page, http://www.nrlmry.navy.mil/aerosol/), these three dust events observed at Nagoya are considered to have originated in East Asian arid regions. Intense dust storms were first observed in the southern Gobi Desert area on 6, 16, and 22 April 2006; subsequently, the dust cloud moved eastward, and reached the middle of Japan after about two or three days.

\subsection{Laboratory and image analytical methods}

After melting re-frozen snow samples, meltwater samples were centrifuged for $10 \mathrm{~min}$, with rotation speed of $3000 \mathrm{rpm}$ at $15^{\circ} \mathrm{C}$, using a centrifuge (KUBOYA 5010). Under this condition, the WIP larger than $0.5 \mu \mathrm{m}$ were separated from the solution. After removing the upper water with a pipette, the remaining WIP were used for further analyses.

The WIP were dried on a clean slide glass in a particle-free air condition, and photographed using an optical microscope (BH2; Olympus Optical Co., Ltd.) equipped with a digital cam- 
era (Moticam 2000; Shimadzu Corp.). The respective magnifications of the digital camera and objective lenses were $20 \times$ and $40 \times$. A calibration slide ( $1 \mathrm{DIV}=0.01 \mathrm{~mm}$; Motic) was used as a sizing ruler. The NIST traceable standard particles of known size (CLINTEX $1560,15.62 \pm 0.24 \mu \mathrm{m}$, JSR) were measured using our system to verify its accuracy for sizing particles. Our results $(15.47 \pm 0.13 \mu \mathrm{m}$ for 32 particles) showed reasonable agreement with the certified value. Parameters of size and shape factors were obtained using image analysis software (Win ROOF; Mitani Corp.). Only particles having diameter greater than $2 \mu \mathrm{m}$ were analyzed to draw a size distribution for the main weight size range of mineral particles. More than 1400 dust particles were analyzed for each sample to provide meaningful statistical information about their shape factors. Our image analysis is based on a two-dimensional cross section of particles. Shape factors addressed in this study include the diameter, circularity factor, and aspect ratio. The particle geometric diameter is defined as a diameter of circle that has the same projection area as the measured particle. The circularity factor is defined as $4 \pi S / l^{2}$, where $S$ is the projection area and $l$ is the periphery length. The particle morphology is a circle, if the circularity factor equals 1 . The aspect ratio is defined as ratio of the longest dimension $b$ to the orthogonal width $a: b / a$. The frequency distributions of these shape factors and their relation with diameters will be discussed later in Section 3 .

\section{Results}

Table 1 summarizes the shape factors of the WIP in the snow samples. Giant WIP larger than $15 \mu \mathrm{m}$ (maximum diameter found in this study was $41 \mu \mathrm{m}$ ) were found in the snow samples, but the frequency of their occurrence was quite low, e.g., one per thousand particles. Observation of giant mineral dust particles $(>75 \mu \mathrm{m})$ has also been reported for aerosol samples of the central north Pacific (Betzer et al. 1988). The median aspect ratios of the WIP were found as 1.22-1.30. The median circularity factors were $0.86-0.93$. The uniform aspect ratios (1.22-1.23) and circularity factors (0.92-0.93) were obtained from the snow layers MD-4, MR-4, and KR-4, thereby supporting the reliability of our measurements, as well as lateral continuity for recording an atmospheric event deposition at the Mt. Tateyama area.

\subsection{Aspect ratio}

Table 1 shows that the median aspect ratios of the WIP in layer $4(1.22-1.23)$ were lower than other layers 1-3 (1.28-1.30). The median aspect ratios (1.28-1.30) in layers 1-3 were slightly smaller than that of 1.4 reported by Okada et al. (1987) for Asian dust event particles at Nagoya; they were also smaller than Okada et al. (2001) for mineral dust particles at source areas in China. The median aspect ratio of particles in layer 4 was much smaller than those obtained at Chinese source regions (Okada et al. 2001).

In our measurements, the water-soluble part of mineral dust particles was dissolved before analysis. Although dissolution of the watersoluble part of dust particles might shrink the particle size to that of the remaining insoluble part of original aerosol particles, the aspect ratio would not change significantly. Wang et al. (2005) analyzed the water-soluble part of dust aerosols in six dust storms at Beijing, China,

Table 1. Summary of shape factors of water-insoluble particles in dusty snow layers at Mt. Tateyama.

\begin{tabular}{lcccc}
\hline Sample & $\begin{array}{c}\text { Number of } \\
\text { analyzed particles }\end{array}$ & $\begin{array}{c}\text { Maximum } \\
\text { diameter }(\mu \mathrm{m})\end{array}$ & $\begin{array}{c}\text { Median Aspect } \\
\text { ratio }\end{array}$ & $\begin{array}{c}\text { Median Circularity } \\
\text { factor }\end{array}$ \\
\hline MR-1 & 2598 & 37.7 & 1.28 & 0.89 \\
MR-2 & 1706 & 41.0 & 1.30 & 0.86 \\
MR-3 & 1400 & 18.2 & 1.29 & 0.87 \\
MR-4 & 2064 & 14.7 & 1.22 & 0.93 \\
KR-4 & 2042 & 14.4 & 1.22 & 0.93 \\
MD-4 & 1691 & 17.6 & 1.23 & 0.92 \\
\hline
\end{tabular}



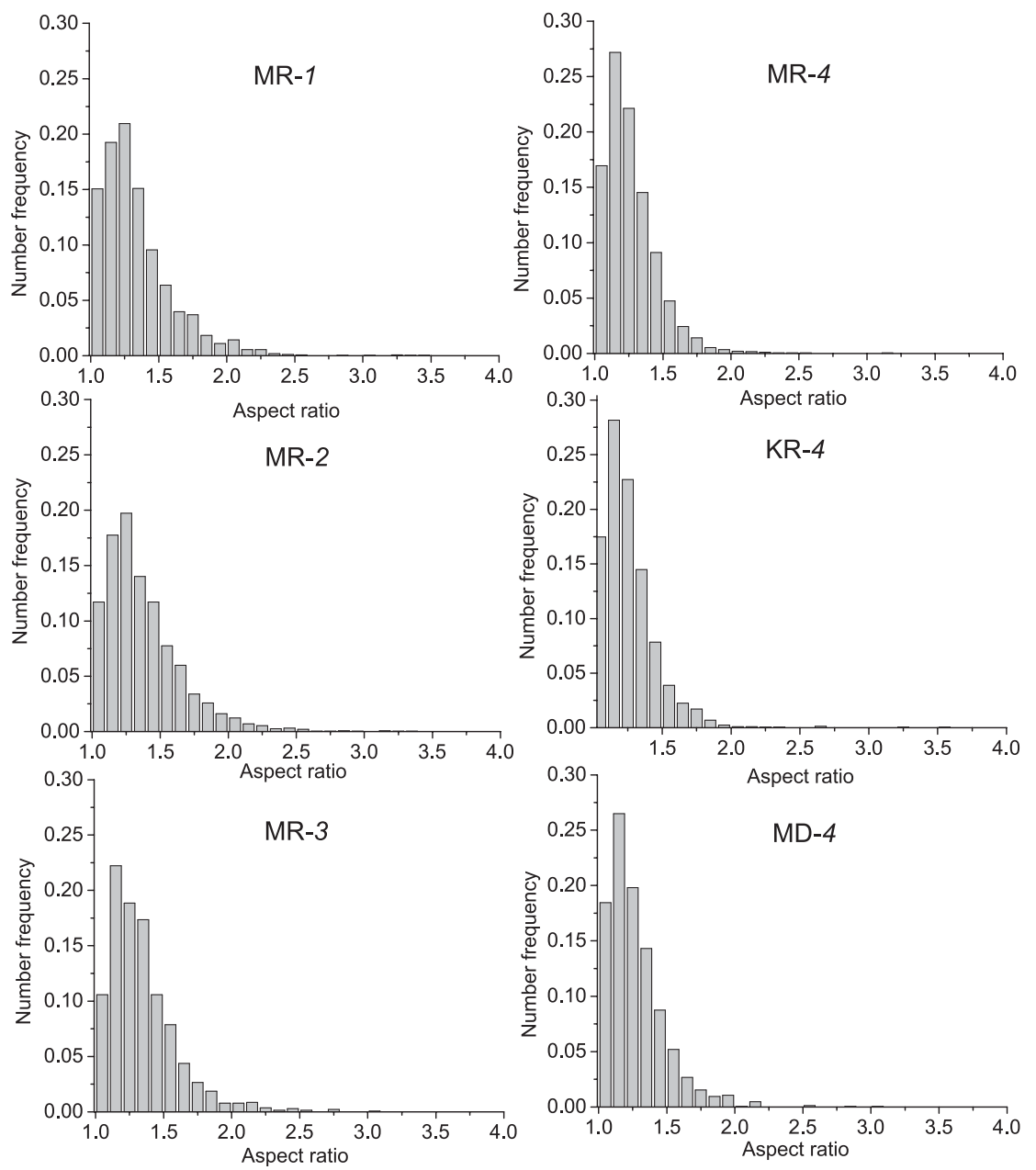

Fig. 3. Frequency distribution of the aspect ratio of the water-insoluble particles in dirty snow layers.

and found that the average mass fraction of water-soluble ionic part in the dust aerosols was only $3.2 \%$ in TSP and $4.7 \%$ in $\mathrm{PM}_{2.5}$ in a super dust storm. Therefore, the size of WIP might not show a great difference from the original aeolian mineral dust. On the other hand, because Okada et al. (2001) measured the radius range as $0.1-6 \mu \mathrm{m}$, differences in the measuring size range might introduce a slight difference for comparison with our data, which will be discussed later in more detail.

Figure 3 shows frequency distributions of the aspect ratio of the WIP. Few particles were found with an aspect ratio greater than 2.5. Overall, $80 \%$ of particles had the aspect ratio in the range of 1.0-1.5 for layers $1-3$, whereas
$90 \%$ of particles in layer 4 fall into the same range, which indicated that the frequency distribution for layer 4 is more concentrated within the narrow range.

\subsection{Circularity factor}

The median circularity factors of WIP were 0.86-0.89 for layers 1-3, and 0.93 for layer 4 (Table 1). Frequency distributions of the circularity factors are shown in Fig. 4. The frequency distributions of the WIP in layer 4 show that $95 \%$ of the WIP fall into the narrow range between 0.8 and 1 , whereas the frequency distributions for the other layers have a broader distribution; 78-84\% of the WIP fall in the same range. 

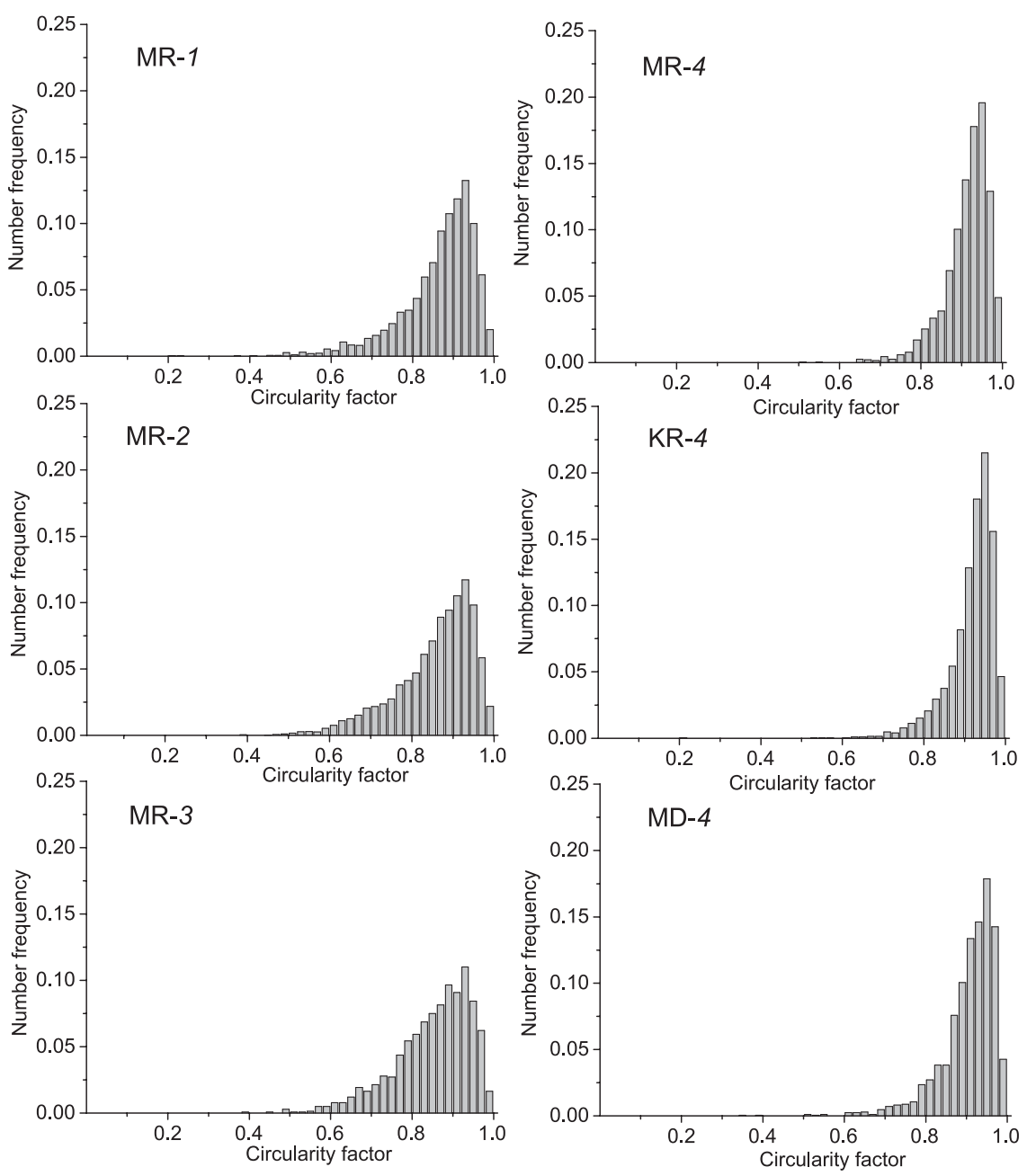

Fig. 4. Frequency distribution of the circularity factor of the water-insoluble particles in dirty snow layers.

\subsection{Relationship of diameter with circularity factors and aspect ratios}

Figure 5 shows box plots of the shape factors of the WIP divided into five size ranges: 2$4 \mu \mathrm{m}, 4-6 \mu \mathrm{m}, 6-8 \mu \mathrm{m}, 8-10 \mu \mathrm{m}$, and $>10 \mu \mathrm{m}$. Differences between maximum and minimum of circularity factor for smallest size $(2-4 \mu \mathrm{m})$ were greater than those for larger size ranges. The median and mean circularity factors of the WIP showed decreasing trends with increasing size range from a value of about 0.9 for $2-4 \mu \mathrm{m}$ to about 0.8 for $>10 \mu \mathrm{m}$. The standard deviation of circularity factor showed no clear difference for sizes less than $8 \mu \mathrm{m}$, but showed large variation for sizes greater than $8 \mu \mathrm{m}$, partly because of the few particles examined. Median and mean aspect ratios of the WIP showed constant values over the size range, which agrees with results reported by Okada et al. (2001), who concluded that the aspect ratios of dust aerosols at Chinese source areas exhibited no clear size dependence.

\section{Discussion}

\subsection{Origin of the WIP in the snow samples}

Figure 6 shows number-size distributions of the WIP in the snow samples and other reference samples. Table 2 lists size parameters fitted with lognormal distributions. The size distribution was fitted to the lognormal distribution optimally with the $r^{2}$ at ca. 0.9999 . The size distributions of MR-4, KR-4, and MD-4 mu- 

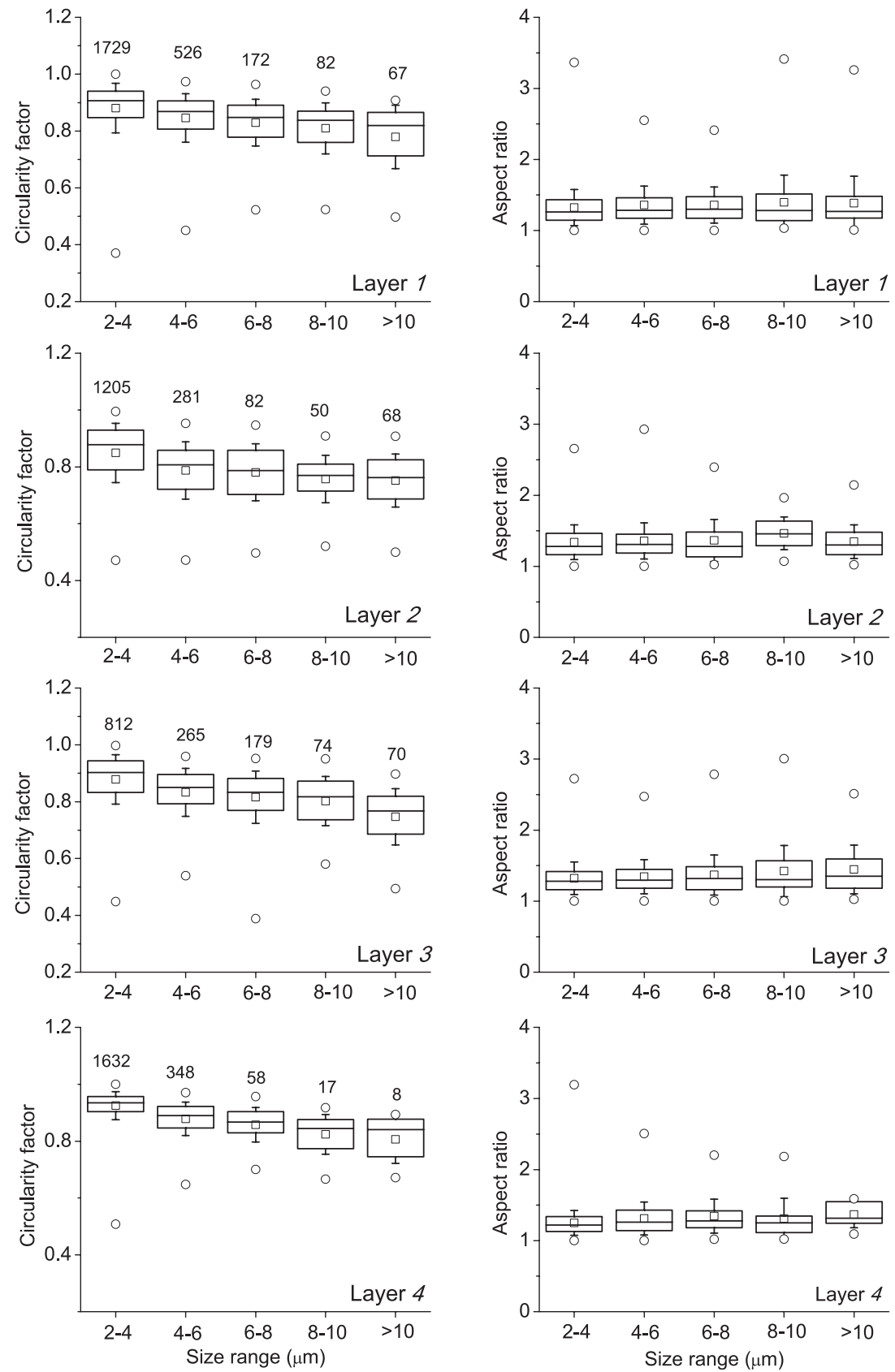

Fig. 5. Statistical box chart for shape factors of water-insoluble particles, classified into different size ranges. The number above the box represents the number of particles measured in the size range. The up and down boundaries of the box respectively indicate the 25th and 75th percentiles, the line within the box marks the median. The square within the box indicates mean. The "O" marks indicate Maximum and Minimum. Whiskers indicate the standard deviation.

tually agreed for modal diameters and standard deviations (shown as $\log \sigma$ ). Mono-modal or bi-modal size distributions were observed for WIP. The median diameters were $1.00-$
$2.23 \mu \mathrm{m}$ for fine mode with $\log \sigma$ of 0.17 0.36 . For layer 3 , an additional coarse mode was also identified with median diameter of $6.35 \mu \mathrm{m}$ with $\log \sigma$ of 0.14 . According to modal 

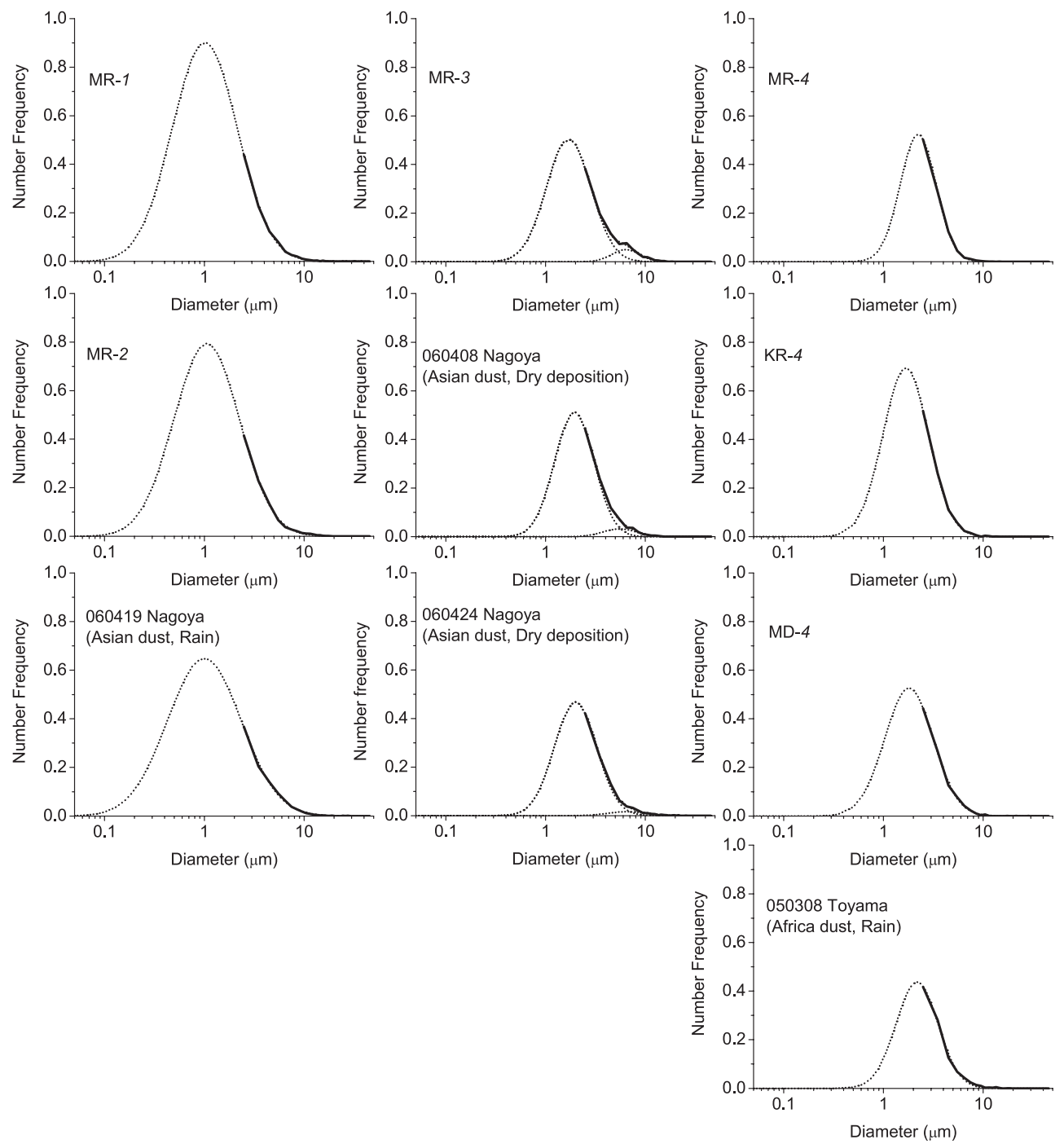

Fig. 6. Number-size distribution of the water-insoluble particles in the dirty snow layers in comparison with reference samples. The thick line represents the measured data. The dotted line represents Gaussian fitting results. Reference samples for Asian dust were collected on 19 April 2006 at Nagoya (rain, 060419), on 8 April 2006 at Nagoya (dry deposition, 060408), and on 24 April 2006 at Nagoya (dry deposition, 060424). The reference for African dust was collected on 8 March 2005 at Toyama (rain, 050308).

characteristics, these size distributions were classified into three groups: (1) layers 1 and 2 as similar median diameters with large $\log \sigma$ (>0.3), (2) layer 3 as the bi-modal distribution of the predominant fine mode with the secondary coarse mode; and (3) layer 4 of moderate median diameter and narrow standard deviation with fewer giant $(>15 \mu \mathrm{m})$ particles.

As shown in Fig. 6, the size distributions of group (1) are similar to that of the WIP in the rain sample obtained at Nagoya on 19 April 2006. The bi-modal size distribution of the WIP as dry-deposited (snow layer 3) agrees well with those of Asian dust events deposited by dry processes at Nagoya on 8 April 2006 and 24 April 2006, implying that the bi-modal distribution of the main fine mode with the secondary coarse mode seems to be a characteristic for the dry deposited Asian dust in this area.

Backward air trajectories (NOAA HYSPLIT 
Table 2. Parameters of number-size distribution fitted to Gaussian equation.

\begin{tabular}{|c|c|c|c|c|c|c|}
\hline \multirow[b]{2}{*}{ Possible source } & \multirow[b]{2}{*}{ Sample } & \multicolumn{2}{|c|}{ Mode 1} & \multicolumn{2}{|c|}{ Mode 2} & \multirow[b]{2}{*}{$R^{2}$} \\
\hline & & D1 & $\log \sigma 1$ & D2 & $\log \sigma 2$ & \\
\hline Asian origin & MR-1 & 1.01 & 0.33 & & & 0.9995 \\
\hline \multirow[t]{2}{*}{ Wet deposition } & MR-2 & 1.05 & 0.33 & & & 0.9991 \\
\hline & 060419 Nagoya (Asian dust, rain) & 1.00 & 0.37 & & & 0.9980 \\
\hline Asian origin & MR-3 & 1.70 & 0.22 & 6.35 & 0.14 & 0.9987 \\
\hline \multirow[t]{2}{*}{ Dry deposition } & 060408 Nagoya (Asian dust, dry) & 1.94 & 0.20 & 5.51 & 0.17 & 0.9995 \\
\hline & 060424 Nagoya (Asian dust, dry) & 1.99 & 0.21 & 6.51 & 0.19 & 0.9996 \\
\hline \multirow[t]{4}{*}{ African origin } & MR-4 & 2.23 & 0.18 & & & 0.9999 \\
\hline & $\mathrm{KR}-4$ & 1.67 & 0.23 & & & 0.9997 \\
\hline & $\mathrm{MD}-4$ & 1.80 & 0.24 & & & 0.9990 \\
\hline & 050308 Toyama (African dust, rain)* & 2.17 & 0.21 & & & 0.9976 \\
\hline
\end{tabular}

D1 and D2 represent mean diameters $(\mu \mathrm{m})$ of lognormal distributions. D1 is an inferred value by fitting which was carried out for the data of number-size distribution of particles more than $2 \mu \mathrm{m}$.

Log $\sigma 1$ and $\log \sigma 2$ respectively indicate logarithms of the standard deviation.

$R^{2}$ indicate the coefficient of fitting.

* The origin was considered on the basis of the research by Park et al. (2005).

model, Draxler and Rolph 2003; Rolph 2003)

(Figs. 7a and 7b) and the visibility reducing surface weather reports for snow layers 2, 3 (http://www.nrlmry.navy.mail/aerosol/), the rain on 19 April, and dry-deposited Asian dust on 8 and 24 April 2006 show that the major source areas of the WIP could be near the Gobi and Taklamakan Desert areas at 1 to several days prior without any noticeable difference between wet and dry deposition at this area.

Surprisingly, mono-modal and narrow characteristics of layer 4 closely resembled those of the Saharan dust sample on March 2005. For the trajectory for layer 4 (Fig. 7c), air parcels ascended from the surface of African arid regions at about 1 week ago, and then stayed at the middle troposphere for a few days. Indeed, a dust storm event was reported at Benina (Libya, $32.8^{\circ} \mathrm{N}, 20.26^{\circ} \mathrm{E}$ ) on 27 December 2000 (JMA, Monthly Report, December, 2000), when the backward trajectory was located within the surface boundary layer. This fact suggests that the WIP in layer 4 probably originated from the Sahara Desert (Fig. 7c).

Some precedent reports have described lognormal size parameters of Asian dust in Japan
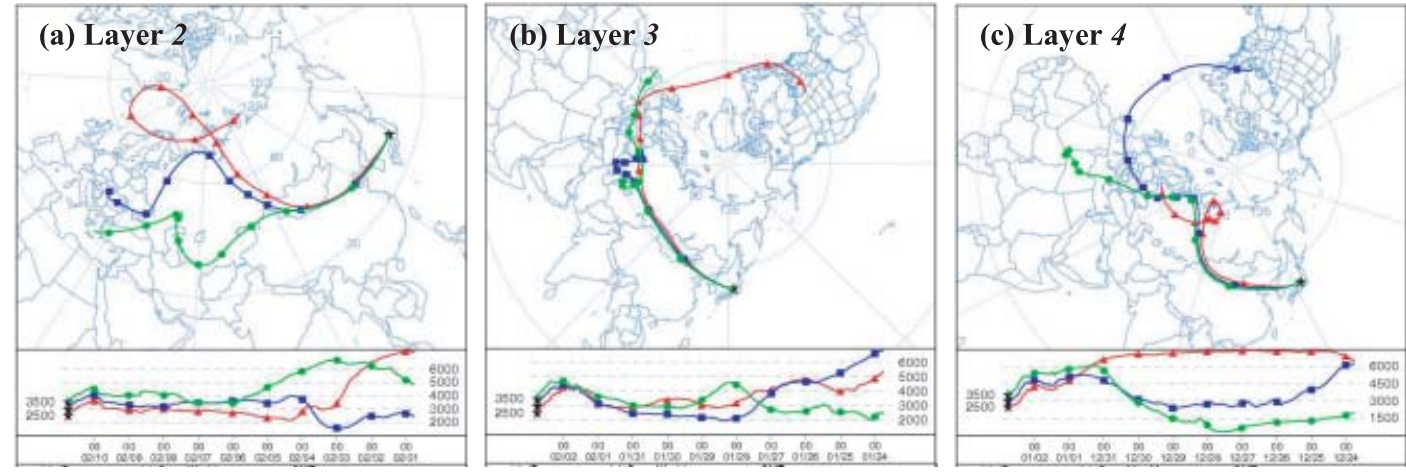

Fig. 7. 10-day backward air trajectories of air parcels reaching Mt. Tateyama, for dust layer 2 deposited on 11 Feb. 2001(a), layer 3 deposited on 3 Feb. 2001(b), and layer 4 deposited on 3 Jan. $2001(\mathrm{c})$. The start point of trajectory was set at $36.60^{\circ} \mathrm{N}, 137.60^{\circ} \mathrm{E}$ (Mt. Tateyama), and altitude at 2500, 3000, and $3500 \mathrm{~m}$ a.s.l. 
(Arao and Ishizaka 1986; Nakajima et al. 1989) and of aeolian dust deposited in the Pacific Ocean (Rea et al. 1995) after long-range transport from Asian source regions. The median diameters in this study (Table 2) agree well with those reports (ca. one to several micrometers).

\subsection{Change of shape factor distributions during long-range transport}

Figure 8 shows frequency distributions of circularity factors and aspect ratios of dust obtained in Chinese source areas (Okada et al. 2001), the Asian dust (layer 2) at Mt. Tateyama, and African dust (layer 4) at Mt. Tateyama. The particle observed in Chinese source areas by Okada et al. (2001) was 0.1$6 \mu \mathrm{m}$ in radius, whereas the particle measured in this study was greater than $2 \mu \mathrm{m}$ diameter. To note differences in analysis of the size between submicrometer and super micrometer ranges, we observed the shape factors of small (0.1-5.78 $\mu \mathrm{m}$ in diameter, Fig. 8 column b) particles using scanning electron microscopy (SEM, S-3000N; Hitachi Ltd.), and compared those observations with data (Fig. 8 column $c$ ) obtained using an optical microscope. As shown in columns $b$ and $c$ of Fig. 8, the distributions of the dust as examined using SEM $(0.1-5.78 \mu \mathrm{m}$ diameter) were slightly flatter and wider than those of dust larger than $2 \mu \mathrm{m}$ in diameter as recorded using the optical microscope. More importantly, the difference between column $a$ and $b$ or $c$, as well as $b$ or $c$ and $d$ is apparent. Thus, the difference of shape factors from Chinese source areas and the WIP in snow layer 2 or 4 might not be derived from the difference of analyzed size range, but might represent the difference in the samples' respective characteristics.

The distributions of the circularity factors $(0.2-1)$ and the aspect ratios (1.0-3.0) in Chinese source areas (Fig. 8 column $a$ ) show wider distributions than Asian dust at Mt. Tateyama (column $b$ and $c$ ). Thus, the dust particles at Chinese source areas have a greater variety of irregular shapes. Data of the African dust (column $d$ ) from Mt. Tateyama show a much narrower distribution: most circularity factors were $0.8-1.0$; most of the aspect ratios were 1.0-1.5. In other words, most African dust collected at Mt. Tateyama was nearly spherical, but slightly elongated particles. We will discuss the possibility of the difference for the shape factors at African source areas, and changes during the transport to Japan, to interpret these differences in the shape factors.

Because mineralogical studies of Saharan dust show that illite-to-kaolinite ratios decrease from west to east (Caquineau et al. 2002), and that Saharan dust contains less calcium than Chinese loess (Kureger et al. 2004), the shape factor distributions might also differ from one source area to another. The aspect ratio distributions of the Saharan dust collected over Puerto Rico (ca. 5000 km from the Sahara Desert)
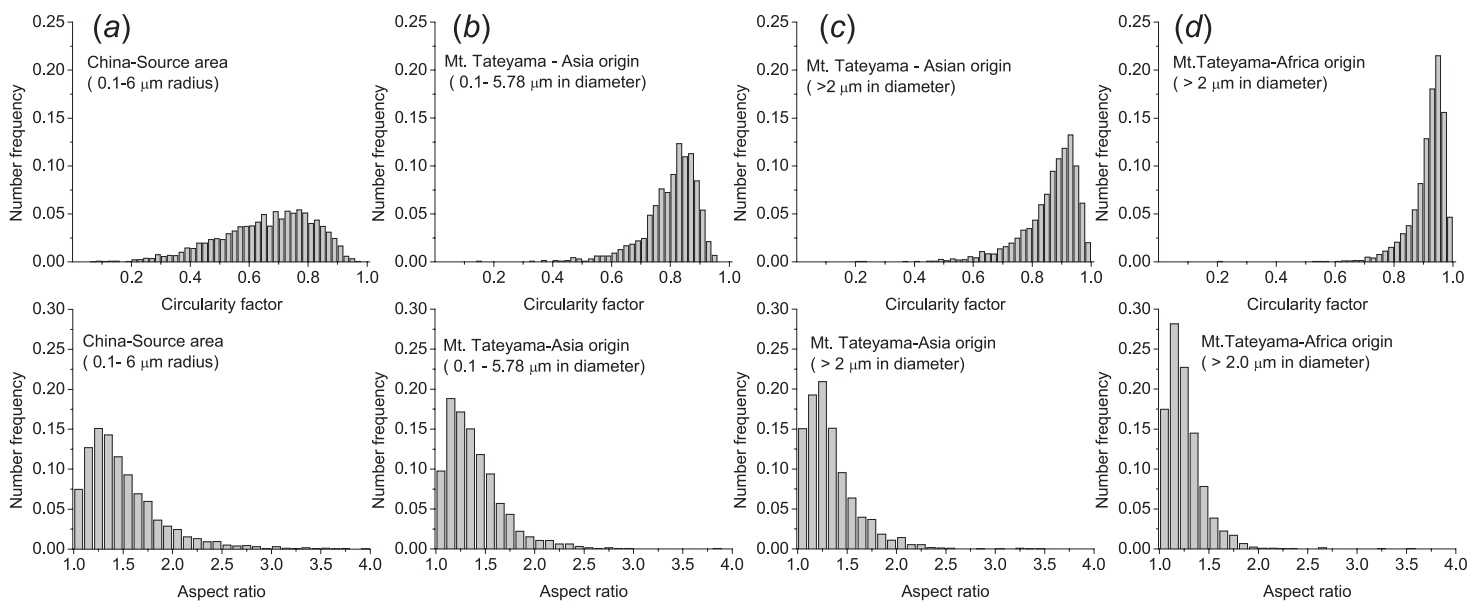

Fig. 8. Distributions of circularity factors and aspect ratios at Chinese source areas (Okada et al. 2001) for column ( $a$ ), dust layer 2 at Mt. Tateyama for columns (b) and (c), dust layer 4 for column $(d)$. 
were almost independent of size, and the distributions did not differ from one sample to another for size $<10 \mu \mathrm{m}$ (Reid et al. 2003). Reid et al. reported a median aspect ratio of 1.9 and sphericity of 0.71 , with much broader distributions than in our observations (Fig. 8 column d) for both parameters.

As shown in Fig. 8, the distributions of the shape factors in Japan seem to be shifted to higher circularity factors and lower aspect ratios, with increasing transportation distance from the source areas, such as inland China (ca. $3500 \mathrm{~km}$ ), and the Sahara (greater than $12,000 \mathrm{~km}$ ). In other words, the proportion of nearly spherical and less-elongated particles was higher in the sample transported a longer distance from the dust source. According to numerical model experiments (Ginoux 2003), changing the particle shape from spherical to spheroidal with an aspect ratio of 2 , imparted little difference in settling velocities. However, when very elongated particles (aspect ratio $=5$ ) were simulated, the settling velocity of nonspherical particles was apparently less than that of spherical particles. Those results were based on the assumption that the ensembles of dust particles were randomly oriented. For realistic dust particles, the center of gravity will be biased to one side of the longest dimension. Spheroidal particles will be oriented to a direction with the heavier side down, if the center of gravity is biased along the longest dimension. This orientation might engender a higher settling velocity for spheroidal particles than for spherical particles having equal volume. In other words, spherical particles might survive longer distances during transport. Because transport of dust particles in the atmosphere will be complicated by the effects of turbulence at higher wind speeds (Aluko and Noll 2006), and because selection or sorting deposition depending on shape factors are not well understand yet, additional field studies, laboratory studies, and numerical experiments will be necessary to elucidate the observed shifts of shape factor distributions.

\section{Summary and concluding remarks}

The size distribution and shape factors of water-insoluble particles (WIP) in four dirty snow layers deposited in 2001 at Mt. Tateyama, Japan were analyzed using scanning electron microscopy and optical microscopy with a digital camera. The salient results of this study can be summarized as follows.

The median aspect ratios of the WIP in the snow samples at Mt. Tateyama varied from 1.22-1.30. Most (80-90\%) particles had aspect ratios of 1.0-1.5; few particles had aspect ratios greater than 2.5. The median circularity factors of the WIP were $0.86-0.93$. More than $78 \%$ of particles had a circularity factor of $0.8-1$. Median and mean circularity factors of the WIP showed decreasing trends with increasing size range from a value of about 0.9 for $2-4 \mu \mathrm{m}$ to about 0.8 for $>10 \mu \mathrm{m}$, whereas the values of the median and mean aspect ratio exhibited no clear size dependence.

Mono-modal or bi-modal size distributions were observed for WIP in the snow samples. The median diameters of number-size distributions were $1-2 \mu \mathrm{m}$ for the predominant mode with $\log \sigma$ of $0.17-0.36$. In addition to the main mode, the secondary coarse mode was identified in the WIP of layer 3, as the median diameter of the coarse mode was $6 \mu \mathrm{m}$ with $\log \sigma$ of 0.14 , which was possibly dry-deposited.

Based on backward air trajectories and visibility reducing surface weather reports, possible source areas of the WIP in the snow samples were suggested as the East Asian arid regions (such as Gobi and Taklamakan Desert areas) for layers 2 and 3, and the Sahara desert for layer 4. Combined with the additional results of other Saharan dust detected in rain (8 March 2005) samples at Toyama and dry deposition dust samples (8 and 24 April 2006) at Nagoya, the number and the shape factor distributions versus particle size were characterized as: (1) narrower distributions of numbersize and shape factors for Saharan dust, and (2) a bi-modal number-size distribution for drydeposited dust.

Comparing the distributions of the shape factors among samples of Chinese source areas, snow layers 2 (Asian dust), and 4 (Saharan dust), it is suggested that the proportion of near spherical (higher circularity) and less elongated (aspect ratio close to 1) particles was higher in the sample transported a longer distance from the source areas of dust. The effect of an unbalanced center of gravity for elongated spheroid particles was suggested to interpret the shift of the shape factors during long-range 
transport. Additional field studies, laboratory studies, and numerical experiments are necessary to understand the shifts of shape factor distributions.

\section{Acknowledgements}

We are grateful to Prof. Y. Iwasaka of Kanazawa University for providing the image software, Dr. M. Kido and Mr. T. Mizoguchi of Toyama Prefectural Environmental Science Research Center for dust samples at Toyama, and Drs. K. Okada and Te. Aoki of the Meteorological Research Institute for numerical data of shape factors at Chinese source areas and for providing reference information. The authors gratefully acknowledge the NOAA Air Resources Laboratory (ARL) for provision of the HYSPLIT transport model website (http:// www.arl.noaa.gov/ready.html) and NRL/ Monterey Aerosol page (http://www.nrlmry .navy.mil/aerosol/) for visibility-reducing surface weather reports used in this publication. This study was partially supported by a Ministry of Education, Culture, Sports, Science and Technology, Grant-in-Aid for Scientific Research (C) 13680601 and (B) 15310012.

\section{References}

Aluko, O. and K.E. Noll, 2006: Deposition and suspension of large, airborne particles. Aerosol Sci. Technol., 40, 503-513.

Arao K. and Y. Ishizaka, 1986: Volume and mass of yellow sand dust in the air over Japan as estimated from atmospheric turbidity. J. Meteor. Soc. Japan, 64, 79-94.

Arimoto, R., R.A. Duce, B.J. Ray, and C.K. Unni, 1985: Atmospheric trace elements at Enewetak Atoll: 2. Transport to the ocean by wet and dry deposition. J. Geophys. Res., 90, 2391-2408.

Astafyeva, L.G. and V.A. Babenko, 2004: Interaction of electromagnetic radiation with silicate spheroidal aerosol particles. J. Quant. Spectrosc. Radiat. Transfer, 88, 9-15.

Betzer, P.R., K.L. Carder, R.A. Duce, J.T. Merrill, N.W. Tindale, M. Uematsu, J.T. Costello, R.W. Young, R.A. Feely, J.A. Breland, R.E. Bernstein, and A.M. Greco, 1988: Long-range transport of giant mineral aerosol particles. Nature, 336, 568-571.

Caquineau, S., A. Audichet, L. Gomes, and M. Legrand, 2002: Mineralogy of Saharan dust transported over north western tropical Atlantic Ocean in relation to source regions. J. Geophys. Res., 107, 4251-4262.
Duce, R.A., C.K. Unni, B.J. Ray, J.M. Prospero, and J.T. Merrill, 1980: Long-range atmospheric transport of soil dust from Asia to the tropical North Pacific: Temporal variability. Science, 209, 1522-1524.

Gao, Y. and J.R. Anderson, 2001: Characteristics of Chinese aerosols determined by individualparticle analysis. J. Geophys. Res., 106, 1803718045.

Ginoux, P., 2003: Effects of nonsphericity on mineral dust modelling. J. Geophys. Res., 108 (D2), doi:10.1029/2002JD002516.

IPCC, 2001: Climate Change 2001: The Scientific Basis (IPCC Third Assessment Report). Houghton, J.T., Ding, Y., Griggs, D.J., Noguer, M., Van Der Linden, P.J., Xiaosu, D. (eds.), Cambridge University Press.

Kahnert, M. and A. Kyling, 2004: Radiance and flux simulations for mineral dust aerosols: Assessing the error due to using spherical or spheroidal model particles. J. Geophys. Res., 109, D09203.

Kahnert, M., T. Nousiainen, and B. Veihelmann, 2005: Spherical and spheroidal model particles as an error source in aerosol climate forcing and radiance computations: A case study for feldspar aerosols. J. Geophys. Res., 110, D18S13, doi:10.1029/2004JD005558.

Kahnert, M. and T. Nousiainen, 2006: Uncertainties in measured and modelled asymmetry parameters of mineral dust aerosol. J. Quant. Spectrosc. Radiat. Transfer, 100, 173-178.

Kalashnikova, O.V. and I.N. Sokolik, 2002: Importance of shapes and compositions of windblown dust particles for remote sensing at solar wavelengths. Geophys. Res. Lett., 29, 1398, 10. 1029/2002GL014947.

Kalashnikova, O.V. and I.N. Sokolik, 2004: Modeling the radiative properties of nonspherical soilderived mineral aerosols, J. Quant. Spectrosc. Radiat. Transfer, 87, 137-166.

Konert, M. and J. Vandenberghe, 1997: Comparison of laser grain size analysis with pipette and sieve analysis: a solution for the underestimation of the clay fraction. Sedimentology, 44, $523-535$.

Krueger, B.J., V.H. Grassian, J.P. Cowin, and A. Laskin, 2004: Heterogeneous chemistry of individual mineral dust particles from different dust source regions: the important of particle mineralogy. Atmos. Environ., 38, 6253-6261.

Masuda, K., Y. Mano, H. Ishimoto, M. Tokuno, Y. Yoshizaki, and N. Okawara, 2002: Assessment of the nonsphericity of mineral dust from geostationary satellite measurements. Remote Sens. Environ., 82, 238-247. 
Naito, M., O. Hayakawa, K. Nakahira, H. Mori, and J. Tsubaki, 1998: Effect of particle shape on the particle size distribution measured with commercial equipment. Powder Technology, 100, 52-60.

Nakajima, T., M. Tanaka, M. Yamano, M. Shiobara, K. Arao, and Y. Nakanishi, 1989: Aerosol optical characteristics in the Yellow sand events observed in May, 1982 at Nagasaki-Part II Models. J. Meteor. Soc. Japan, 68, 279-291.

Okada, K., A. Kobayashi, Y. Iwasaka, H. Naruse, T. Tanaka, and O. Nemoto, 1987: Features of individual Asian dust-storm particles collected at Nagoya, Japan. J. Meteor. Soc. Japan, 65, $515-521$.

Okada, K., J. Heintzenberg, K. Kai, and Y. Qin, 2001: Shape of atmospheric mineral particles collected in three Chinese arid-regions. Geophys. Res. Lett., 28, 3123-3126.

Osada, K., H. Iida, M. Kido, K. Matsunaga, and Y. Iwasaka, 2004: Mineral dust layers in snow at Mount Tateyama, central Japan: formation processes and characteristics. Tellus, 56B, 382-392.

Park, C.B., N. Sugimoto, I. Matsui, A. Shimizu, B. Tatarov, A. Kamei, C.H. Lee, I. Uno, T. Takemura, and D.L. Westphal, 2005: Long-range transport of Saharan dust to East Asia observed with lidars. SOLA, 1, 121-124.

Rea, D.K. and S.A. Hovan, 1995: Grain size distribution and depositional processes of the mineral component of abyssal sediments; Lessons form the North Pacific. Paleoceanography, 10, 251258.

Reid, E.A., J.S. Reid, M.M. Meier, M.R. Dunlap, S.S. Cliff, A. Brounas, K. Perry, and H. Maring, 2003: Characterization of African dust transported to Puerto Rico by individual particle and size segregated bulk analysis. J. Geophys. Res., 108 (D19), doi:10.1029/2002JD2935.

Sakai, T., T. Shibata, S.A. Kwon, Y.S. Kim, K. Tamura, and Y. Iwasaka, 2000: Free tropospheric backscatter, depolarization ratio, and relative humidity measured with the Raman lidar at Nagoya in 1994-1997: Contributions of aerosols from the Asian Continent and the Pacific Ocean. Atmos. Environ., 34, 431-442.

Sokolik, I.N. and O.B. Toon, 1996: Direct radiative forcing by anthropogenic airborne mineral aerosols. Nature, 381, 681-683.

Sokolik, I.N., D.M. Winker, G. Bergametti, D.A. Gillette, G. Carmichael, Y.J. Kaufman, L. Gomes, L. Schuetz, and J.E. Penner, 2001: Introduction to special section: Outstanding problems in quantifying the radiative impacts of mineral dust. J. Geophys. Res., 106(D16), 1801518028, 10.1029/2000JD900498.

Uematsu, M., R.A. Duce, J.M. Prospero, L. Chen, J.T. Merrill, and R.L. McDonald, 1983: Transport of mineral aerosol from Asia over the North Pacific Ocean. J. Geophys. Res., 88, 5343-5352.

Volken, M. and T. Schumann, 1993: A critical review of below-cloud aerosol scavenging results on Mt. Rigi. Water, Air, and Soil Pollut., 68, 1528.

Wang, Y., G.S. Zhuang, Y.L. Sun, and Z.S. An, 2005: Water-solution part of the aerosol in the dust storm season-evidence of the mixing between mineral and pollution aerosols. Atmos. Environ., 39, 7020-7029.

Yamamoto, N., Y. Shinozuka, K. Kumagai, M. Fujii, and Y. Yanagisawa, 2004: Particle size distribution quantification by microscopic observation. J. Aerosol Sci., 35, 1225-1234. 